\title{
Contribution of the Walsh Orbitals to the Excited States of Conjugated Cyclopropyl Molecules (A PMO Treatment)
}

\author{
Muthana Shanshal \\ Department of Chemistry, College of Science, University of Baghdad, Adhamiya, Baghdad, Iraq
}

(Z. Naturforsch. 31 a, 488-493 [1976] ; received November 13, 1975)

\begin{abstract}
The molecular orbital energies of some conjugated cyclopropyl derivatives are evaluated using 2nd order perturbation. The basis includes the Hückel MOs of the conjugated segment and the Walsh MOs of cyclopropane. The obtained MO sequence is used to discuss the UV absorption spectra of the molecules. It is found that the absorption bands of most of these molecules correspond to $\Delta \cdot \pi^{*}$ or $\Delta-\Delta^{*}$ excitations and not to $\pi-\pi^{*}$ transitions as had been assumed.
\end{abstract}

\section{Introduction}

It has been shown recently that the ground state reactivity of conjugated cyclopropyl derivatives may be discussed successfully in terms of a second order perturbation treatment ${ }^{1}$. The interaction energy between the cyclopropane ring and the conjugated moiety was calculated using the Walsh molecular orbitals of the ring and the Hückel $\pi$-molecular orbitals (MOs) as basis. The extension of this method to the discussion of the electronic configuration of the lowest excited states of such molecules requires the calculation of the energy change for each MO rather than the direct calculation of the total interaction energy between both segments. The MO energy change is given by the equation ${ }^{2}$

$$
\Delta \varepsilon_{\mathrm{u}}=\sum^{(\mathrm{R})}{ }_{\mathrm{v}} a_{\mathrm{u}, \mathrm{s}} b_{\mathrm{v}, \mathrm{r}} \beta_{\mathrm{rs}}+\sum^{(\mathrm{R})} \frac{a_{\mu, \mathrm{s}}^{2} b_{\mathrm{v}, \mathrm{r}}^{2} \beta_{\mathrm{rs}}^{2}}{\varepsilon_{\mathrm{u}}-\varepsilon_{\mathrm{v}}} .
$$

The first term of the equation represents the first order perturbation of the MO $\mathrm{u}$ on segment $\mathrm{S}$ by the MOs v on segment R. It has a significant value in the case of degeneracy between the MO $\mathrm{u}$ in $\mathrm{S}$ and the MO $\mathrm{v}$ in $\mathrm{R}^{2}$. The second term represents the second order perturbation. In our treatment we use the same assumption concerning the equality of $\beta_{\mathrm{C}-\mathrm{C}}\left(\right.$ Walsh MOs) and $\beta_{\mathrm{C}-\mathrm{C}}\left(\right.$ Hückel MOs) ${ }^{1}$. A similar assumption has been recently used for the discussion of the photoelectron spectra of conjugated cyclopropyl molecules ${ }^{3}$. In the case of degeneracy between a $\mathrm{MO} \mathrm{u}$ in $\mathrm{S}$ and a $\mathrm{MO} \mathrm{v}$ in $\mathrm{R}$ the 1st order perturbation was evaluated first and the assignment of the energetically shifted MOs to the interacting segments was done considering the total interaction energy including the 2 nd order term. The correct assignment should yield the bigger interaction energy. The total interaction energy was then summed according to the expression

$$
\Delta E_{\mathrm{R}-\mathrm{S}}=\sum_{\mathrm{u}}^{\text {occ.(s) }} n_{\mathrm{u}} \Delta \varepsilon_{\mathrm{u}}+\sum_{n_{\mathrm{v}}}^{\text {oce.(R) }} n_{\mathrm{v}} \Delta \varepsilon_{\mathrm{v}},
$$

$n_{\mathrm{v}}, n_{\mathrm{u}}=$ electron occupation numbers of the MOs $\mathrm{u}$ and $\mathrm{v}$.

\section{Absorption Spectra of Conjugated Cyclopropyl Derivatives}

The intensive UV absorption bands of the conjugated cyclopropyl molecules have been usually attributed to the $\pi-\pi^{*}$ transition of the conjugated moiety ${ }^{4-13}$. Kasha et al. ${ }^{14}$ showed, however, that the gas phase spectrum of the methyl, cyclopropyl ketone includes a $\Delta-\pi^{*}$ charge transfer band in the region $170-200 \mathrm{~m} \mu$. CNDO calculations confirmed the assumed nature of the bands ${ }^{14}$. A similar $\Delta-\pi^{*}$ band of $225-275 \mathrm{~m} \mu$ was detected for the nonbisected 1, methyl, cyclopropyl, methyl ketone in $n$-hexane solution ${ }^{15}$. The origin of the band was revealed on the basis of a similar PMO treatment as the one reported in this text.

In the following text the elucidation of the electronic configuration for the lowest excited states of such molecules is attempted. The formerly discussed perturbation treatment is utilized.

\section{Vinyl, Cyclopropane}

The absorption spectra of a series of alkyl substituted vinyl, cyclopropanes were measured by Heathcock and Poulter ${ }^{11}$. The reported bands in the region $189-200 \mathrm{~m} \mu$ were attributed to $\pi-\pi^{*}$ transitions of the vinyl group. A systematic hypsochromic shift was observed as a result of introducing a methyl group in the $\mathrm{C}_{1}$ position of the cyclopropyl ring (Table 1). Compared with the ab- 
Table 1. Experimental $\lambda_{\max }$ and $\log \varepsilon$ values for some vinyl cyclopropanes $^{11}$ and $\beta$, cyclopropyl, acrylic esters ${ }^{12}$.

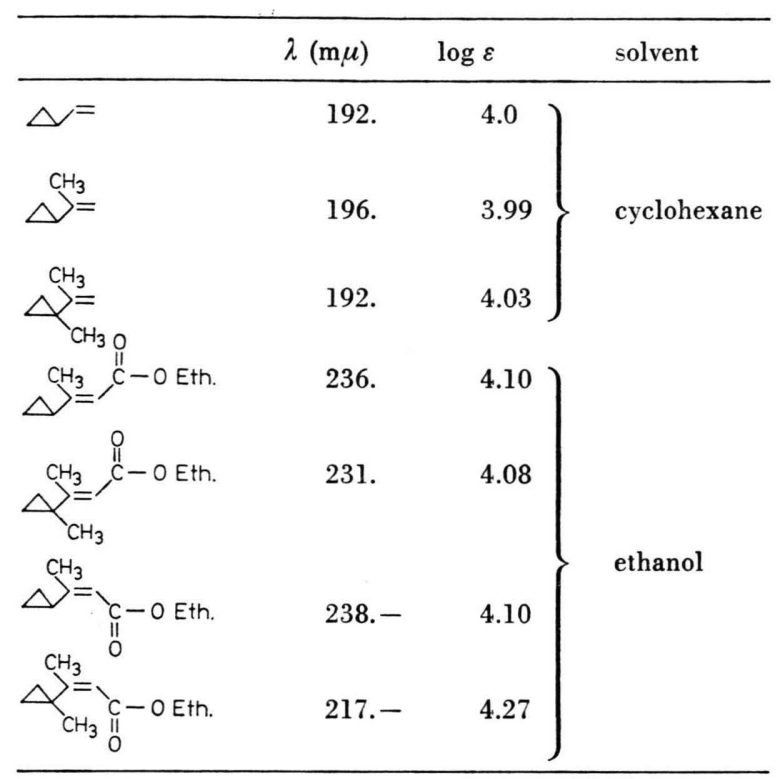

sorption spectra of the open chain olefines a typical bathochromic shift was observed. The perturbation calculation was carried out for the bisected, $\mathrm{C}_{\mathrm{s}}$, conformation of the vinyl, cyclopropyl system. This conformation is known to be favoured in the ground state of the molecule ${ }^{16}$. The obtained orbital sequence as well as the MO energies of the initial segments, ethylene and cyclopropane, are drawn in Figure 1. The cyclopropyl MOs are designated external MOs $\left(\Delta_{\text {ext }}\right)$ and internal MOs $\left(\Delta_{\text {int }}\right)$ instead of our recent $\sigma$ and $\pi$ nomenclature ${ }^{1}$. They are classified relative to the only plane of symmetry of the molecule (Figure 1).

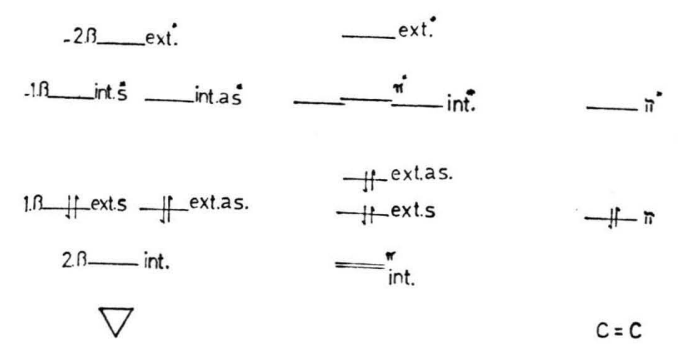

Fig. 1. The molecular orbitals and MO energies of the bisected vinyl cyclopropane as obtained from the vinyl $\pi$-MOs (left) and cyclopropane Walsh MOs (right) through a second order perturbation treatment.
Due to the degeneracy of the bonding MO of ethylene and the $\Delta_{\text {ext }}$ MOs first order perturbation was considered. The $\Delta_{\text {int }}$ MOs and the occupied symmetric $\Delta_{\text {ext }}$ remained unperturbed due to the orthogonality of the first to the MO of the vinyl group and the vanishing $\mathrm{MO}$ coefficient at $\mathrm{C}_{1}$ of the last ${ }^{1}$.

The obtained MO sequence suggests that the highest occupied MO is the antisymmetric external MO $\left(\Delta_{\text {ext.as }}\right)$. The vinyl MO is strongly stabilized as a result of the interaction. The sequence disagrees with that obtained for the same molecule on the bases of a lst order perturbation alone ${ }^{3}$. The lowest unoccupied MOs are the two degenerate and antibonding $\Delta_{\mathrm{int}}^{*}$ MOs. Their energy difference to the weakly destabilized $\pi^{*}$ MO is very small $(+0.07 \beta)$. Thus, it is quite difficult to predict exactly the nature of the lowest unoccupied MO from Fig. 1, given that such a simplified model does not include the electronic exchange and repulsion energies. Symmetry argumentations show that both $\Delta_{\text {ext. as }}-\Delta_{\text {int. as }}^{*}$ and $\Delta_{\text {ext. as }}-\pi^{*}$ transitions are allowed. It may be concluded then that the observed bands in the spectra of vinyl, cyclopropanes are due to $\Delta-\Delta^{*}$ or $\Delta-\pi^{*}$ and not to $\pi-\pi^{*}$ transitions as assumed ${ }^{11}$.

Interesting is the discussion of the hypsochromic shifts resulting from the introduction of an alkyl group in $\mathrm{C}_{1}$ of the ring. The construction of molecular models for the experimentally studied 1 , methyl, vinyl, cyclopropanes shows that no significant steric hinderance should force the molecule towards a planar conformation. The shift should then be due to a hyperconjugative interaction alone. Figure 2 shows the mode of interaction between a $\Delta_{\text {ext. }}$ MO and a methyl group. To assure maximal hyperconjugation the $\mathrm{p}_{z}$ atomic orbital of the $\mathrm{C}_{\mathrm{h}}$ atom is set parallel to the $\mathrm{p}_{z} \mathrm{AO}$ at $\mathrm{C}_{1}$. Among the

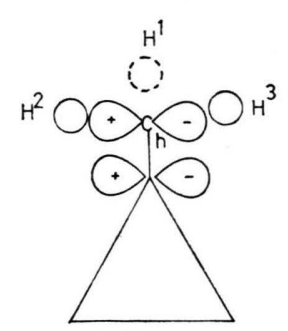

Fig. 2. Hyperconjugative interaction between the $\mathrm{CH}_{3}$ group and a neighbored $\Delta$ ext. MO. 
three orthogonal combinations of the $3 \times \mathrm{H}$ group ${ }^{17}$

$$
\begin{aligned}
& \psi_{\mathrm{h}, 1}=1 / \sqrt{3}\left(\varphi_{\mathrm{H}, 1}+\varphi_{\mathrm{H}, 2}+\varphi_{\mathrm{H}, 3}\right), \\
& \psi_{\mathrm{h}, 2}=1 / \sqrt{6}\left(2 \varphi_{\mathrm{H}, 1}-\varphi_{\mathrm{H}, 2}-\varphi_{\mathrm{H}, 3}\right), \\
& \psi_{\mathrm{h}, 3}=1 / \sqrt{2}\left(\varphi_{\mathrm{H}, 2}-\varphi_{\mathrm{H}, 3}\right),
\end{aligned}
$$

only the 3rd has a positive overlap with the $\mathrm{C}_{\mathrm{h}, \mathrm{p} z} \mathrm{AO}\left(\varphi_{\mathrm{H}, 1} \text { is orthogonal to } \mathrm{C}_{\mathrm{h}, \mathrm{pz}}\right)^{18}$. This combination however corresponds to an antibonding combination of the $\mathrm{H}_{1 \mathrm{~s}}$ AOs with a high energy. Its interaction with the adjacent $\mathrm{C}_{\mathrm{h}, \mathrm{p} z} \mathrm{AO}$ should cause a weak stabilization of this orbital and a small increase in its own energy:

$$
\begin{aligned}
\Delta E_{\mathrm{Ch}, \mathrm{p}_{z}}=\frac{1.1 \beta^{2}(\mathrm{C}-\mathrm{H})}{E_{\mathrm{p} z}-\left(-E_{\mathrm{h}, 3}\right)}, \\
\Delta E_{\mathrm{h}, 3}=\frac{1.1 \beta^{2}(\mathrm{C}-\mathrm{H})}{-E_{\mathrm{h}, 3}-E_{\mathrm{p} z}} .
\end{aligned}
$$

Consequently, the newly formed $\mathrm{C}_{\mathrm{h}, \mathrm{p} z}^{\prime}$ orbital is energetically still higher than the adjacent $\Delta_{\text {ext.as }}$ MO. Their interaction should stabilize the latter and increase the energy of the former orbital.

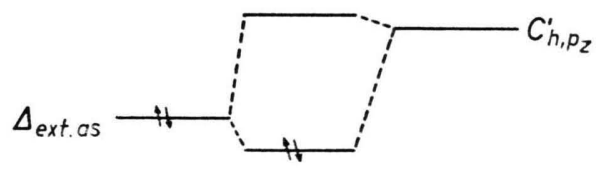

However hyperconjugation has no stabilization influence on the vaccant $\Delta_{\text {int }}^{*}$ or $\pi^{*}$ MO due to their vanishing coefficients on $\mathrm{C}_{1}$. The difference in interaction between the $\mathrm{CH}_{3}$ group and the highest occupied as well as the lowest unoccupied MO causes the observed hypsochromic shift of the band, according to the model.

\section{1-Butadienyl, Cyclopropane}

The UV absorption spectrum of this compound was measured by Julia et al. ${ }^{8}\left(\lambda_{\max }=241 \mathrm{~m} \mu, \log \varepsilon\right.$ $=4.40$, in ethanol). The absorption band was attributed to a $\pi \cdot \pi^{*}$ transition. The 2nd order perturbation confirms this result (Figure 3 ). In this figure it is seen that the highest occupied $\pi$-MO of butadien is destabilized while its lowest unoccupied MO remains energetically almost unchanged.

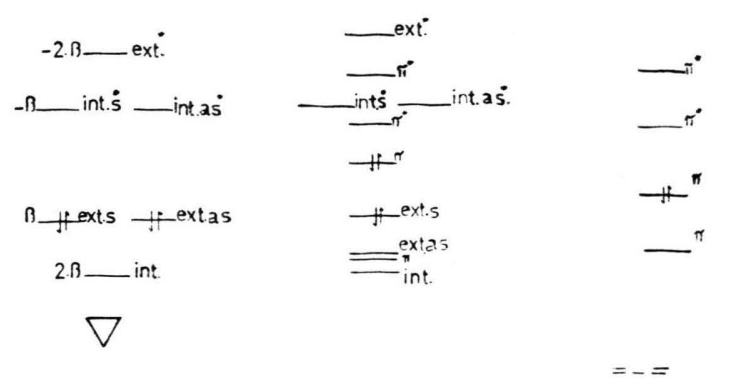

Fig. 3. Same as Fig. 1, but for the bisected 1,butadienyl cyclopropane.

\section{Carbonyl, Cyclopropane}

The absorption spectrum of the methyl, cyclopropyl ketone was reported by different research groups ${ }^{19}$. In all cases the $\mathrm{n}-\pi^{*}$ band was reproduced $\left(\lambda_{\max }=277.5 \mathrm{~m} \mu, \log \varepsilon=1.26\right.$ in $\mathrm{n}$-hexane $)$. Kasha et al. ${ }^{14}$ identified for the same ketone in the gas phase a second and more intensive band the center of which falls approximately at $175 \mathrm{~m} \mu$. It was attributed to an electronic $\Delta-\pi^{*}$ transition. The second order perturbation treatment of the carbonyl, cyclopropane system with a bisected conformation yielded the orbital diagram of Figure 4 . The loca-

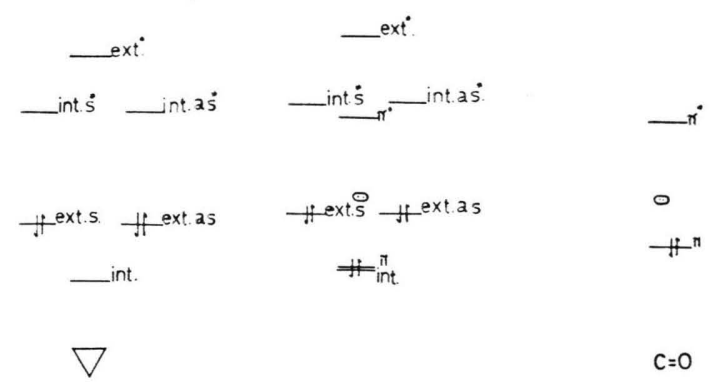

Fig. 4. Same as Fig. 1, but for the bisected carbonyl cyclopropane system.

tion of the $\mathrm{n}-\mathrm{MO}$ is based on a recent photoelectron spectroscopic measurement ${ }^{20}$. According to the diagram the $\pi^{*}$ MO is destabilized and the energy of the $\mathrm{n} \mathrm{MO}$ is not changed by the interaction. The increased energy difference between the $n$ and $\pi^{*}$ MOs explains the hypsochromic shift of these bands in the cyclopropyl ketones relative to those in acetone $\left(\lambda_{\max }=280\right.$ in $n$-hexane $)$. The change in the energy of the $\Delta_{\text {ext. as }}$ MO is negligible. The calculated $\Delta-\pi^{*}$ excitation energy is $1.74 \beta$.

The rotation of the $\mathrm{C}=\mathrm{O}$ group towards the planar conformation causes a stabilization of the $\pi^{*}$ MO and a destabilization of the $\pi$-MO (Fig. 5) 


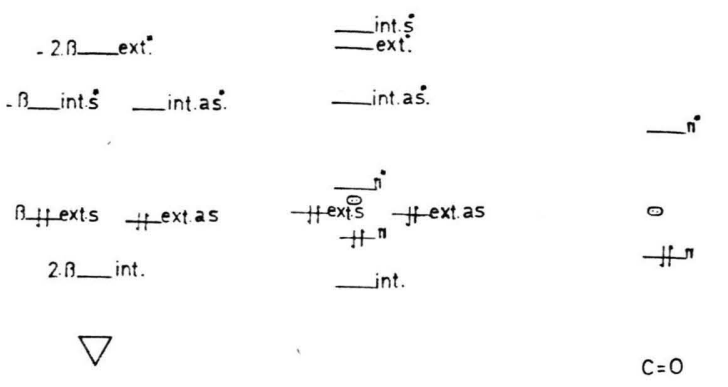

Fig. 5. Same as Fig. 1, but for the planar carbonyl cyclopropane system.

and consequently a bathochromic shift in both $n \cdot \pi^{*}$ and $\Delta_{\text {ext. as }}-\pi^{*}$ bands. The shift was detected for both bands in the spectrum of 1, methyl,cyclopropyl, methyl ketone in $\mathrm{n}$-hexane $\left(\lambda_{\mathrm{n}-\pi^{*}}=282.5 \mathrm{~m} \mu\right.$; $\left.\lambda_{\Delta-\pi^{*}}=220-272 \mathrm{~m} \mu\right)^{21}$. The fact that a $\mathrm{C}_{1}$ methylation of this ketone causes a bathochromic rather than a hypsochromic shift, as in the case of vinyl, cyclopropane, indicates a deformation of the ketone from the bisected to the planar conformation. The change in conformation was concluded on the bases of MINDO/2 calculations also ${ }^{21}$.

\section{$\beta$, Cyclopropyl, Acrylic Esters}

The absorption spectrum of the unalkylated spectrum was measured by Jorgenson and Leung ${ }^{12}$ $\left(\lambda_{\max }=232 \mathrm{~m} \mu, \log \varepsilon=4.2\right.$ in ethanol). The high value of $\varepsilon$ excludes the $n-\pi^{*}$ assignment of the band. The second order MO levels are plotted in Figure 7. It is seen that the perturbation raises the $\Delta_{\text {ext. as }}$ MO and lowers all the occupied $\pi$-MOs of the acrylic group. The lowest unoccupied $\pi^{*}$ MO is slightly destabilized. In the diagram the n-MO is placed lower than the $\Delta_{\text {ext. as }} \mathrm{MO}$, since, the observed and highly intensive band should correspond to a symmetrically allowed rather than an $n-\pi^{*}$ transition.

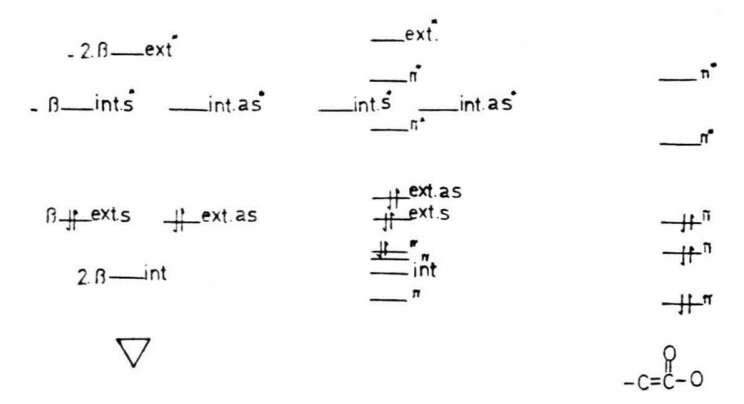

Fig. 6. Same as Fig. 1, but for the bisected $\beta$,cyclopropyl,acrylic ester.
Both symmetry considerations and the orbital sequence (Fig. 6) indicate that the observed band is due to a $\Delta-\pi^{*}$ transition and not to a $\pi-\pi^{*}$ transition. Introducing a methyl group in $\mathrm{C}_{1}$ caused a hypsochromic shift in the absorption band of the esters $^{12}$ (Table 1). A study of the molecular model of the $\mathrm{C}_{1}$, methyl, acrylic esters showed that the bisected conformation is sterically not hindered. The shift is similar then to that in the vinyl, cyclopropanes and may be explained using the same argumentations.

\section{2,(4), Pyridyl, Cyclopropane}

The high values of the extinction coefficients of the longest absorption bands of both ortho and para pyridyl cyclopropanes $\left(\lambda_{\max }=268.5 \mathrm{~m} \mu, \log \varepsilon=3.6\right.$ and $\lambda_{\max }=259 \mathrm{~m} \mu, \log \varepsilon=3.38$ respectively) exclude the $n-\pi^{*}$ assignement for them. The second order perturbation yielded the MO sequence of Figs. 7 and 8. Both molecules were considered to have bisected geometries. In both cases the highest occupied MO is a destabilized $\Delta_{\text {ext. as }}$ MO. The lowest unoccupied MO is a MO of the pyridyl segment. According to the same model the absorption band is due to a $\Delta \cdot \pi^{*}$ transition in both molecules.

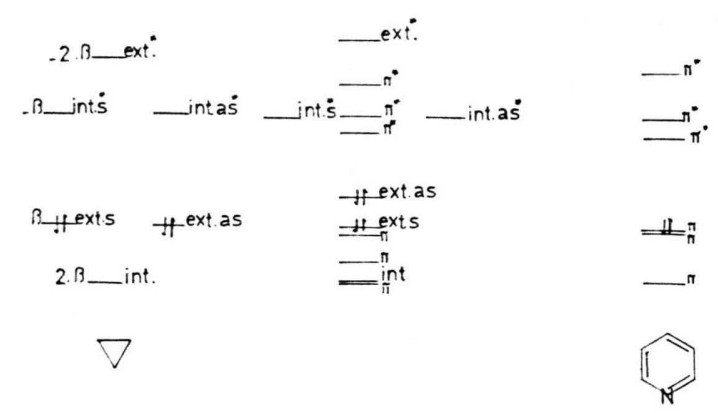

Fig. 7. Same as Fig. 1, but for the bisected 2,pyridyl cyclopropane.

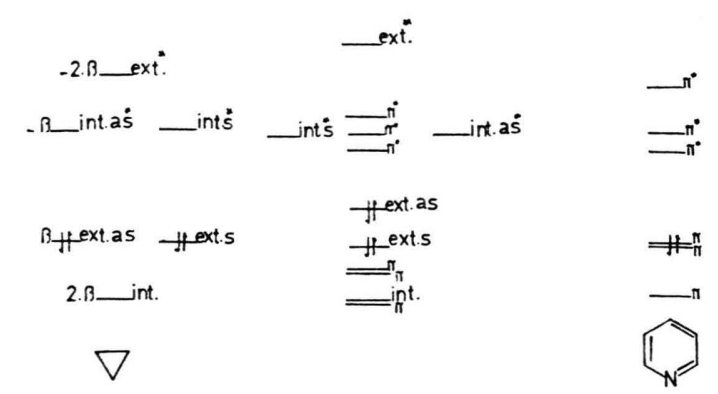

Fig. 8. Same as Fig. 1, but for the bisected 4,pyridyl cyclopropane. 


\section{Phenyl, cyclopropane}

The longest absorption band of $274 \mathrm{~m} \mu$ ( $\log \varepsilon$ $=2.19$ in $\mathrm{EtOH}$ ) was measured by Rogers ${ }^{4}$ and attributed to the perturbed $\pi-\pi^{*}$ transition of benzene ${ }^{22}$. The second order perturbation treatment for its bisected conformation yielded the MO energy levels of Figure 9. It is seen that the highest oc-

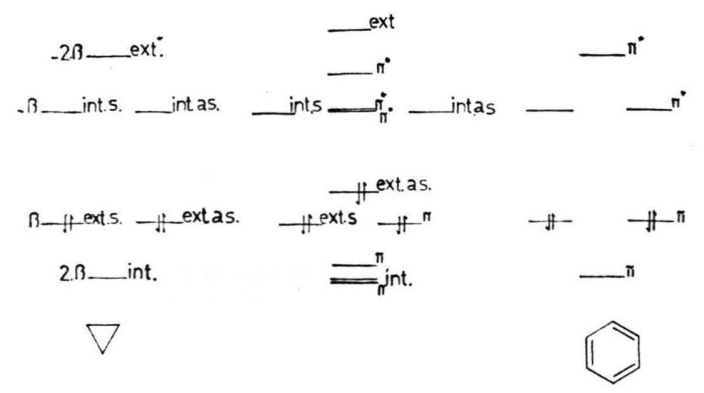

Fig. 9. Same as Fig. 1, but for the bisected phenyl cyclopropane.

cupied MO of benzene is stabilized to $1.74 \beta$ and that the energy of $\Delta_{\text {ext. as }}$ MO of cyclopropane is raised to $0.41 \beta$. The other two degenerate MOs of benzene and cyclopropane are unperturbed due to their vanishing coefficients at the interaction site. Symmetry considerations suggest either the $\Delta_{\text {ext. as }}-\pi^{*}$ or the $\Delta_{\text {ext. as }} \Delta^{*}{ }_{\text {int. as }}$ transition for the longest absorption band. Due to the small difference in the energies of the two excited MOs no definite conclusion about the nature of the band can be drawn from the present treatment. Recently Effenberger et al. ${ }^{23}$ showed that $\lambda_{\max }$ and the longest vibronic band in the absorption spectrum of 1 , methyl, 1, phenyl cyclopropane, with a planar geometry, have approximately the same lengths as those of the bisected phenyl cyclopropane ${ }^{4}$. No significant hypsochromic shift due to the orthogonalization of the benzene $\pi$ MOs to the external Walsh MOs was observed, as would be expected from the Music and Matsen's treatment ${ }^{22}$. The present treatment however, yields equal values for the transition energy in both conformations, suggesting equivalent wave lengths (Figure 10). The PMO treatment suggests

1 M. Shanshal, Z. Naturforsch. 27 a, 1665 [1972].

2 See M. J. S. Dewar, The Molecular Orbital Theory of Organic Chemistry, McGraw-Hill Book Co., New York 1969.

3 E. Heilbronner, R. Gleiter, T. Hoshi, and A. de Meijere, Helv. Phys. Acta 56, 1594 [1973].

4 M. T. Rogers, J. Amer. Chem. Soc. 69, 2544 [1947].

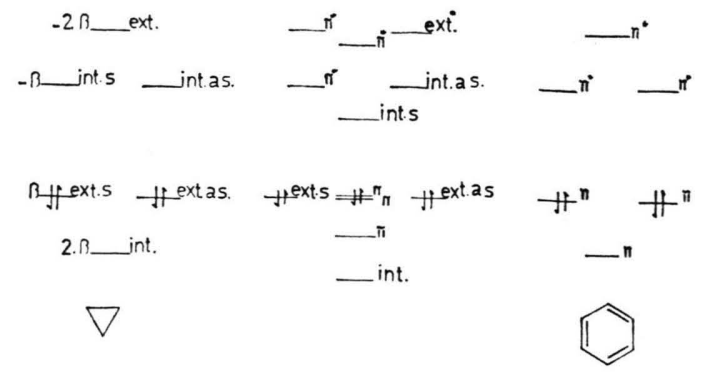

Fig. 10. Same as Fig. 1, but for the planar phenyl cyclopropane.

a $\Delta-\pi^{*}$ and a $\pi-\Delta^{*}$ type transition for the observed bands of the bisected and planar conformations respectively.

Figure 11 shows the correlation of the calculated excitation energies to the experimental wave lengths. With phenyl, cyclopropane showing the biggest deviation from the correlation line, the correlation is acceptable given that the spectra were measured in different solvents.

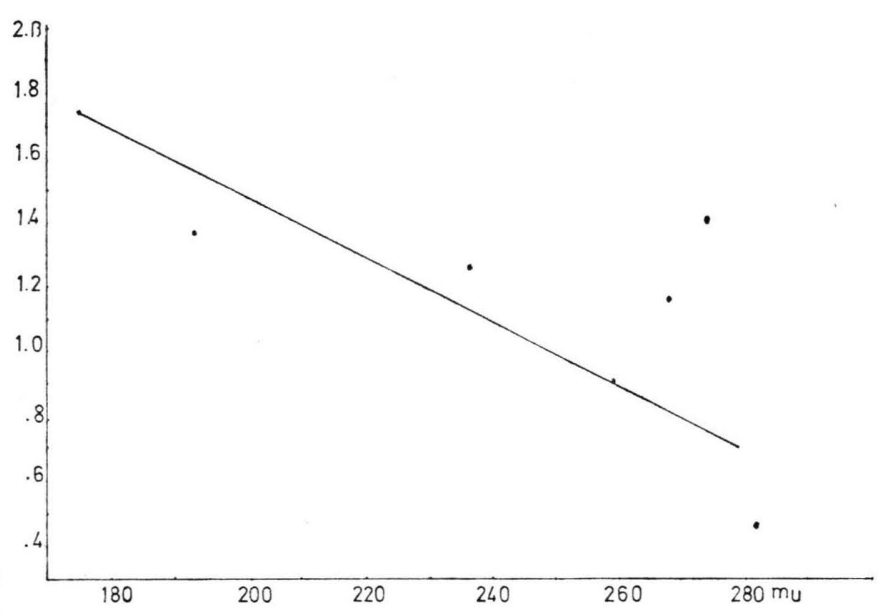

Fig. 11. The correlation of the calculated excitatino energies (PMO treatment) to the experimentally measured wave lengths.

\section{Acknowledgement}

The author thanks Miss M. Mammu and J. A. AlKhafaji for their calculation assistance.

5 A. L. Goodman and R. H. Eastman, J. Amer. Chem. Soc. 86, 908 [1964].

6 L. A. Strait, R. Ketcham, D. Jambotkar, and V. P. Shah, J. Amer. Chem. Soc. 86, 4628 [1964].

7 S. Julia, M. Julia, S.-Y. Tchen, and P. Graffin, Bull. Soc. Chim. France 1964, 3218. 
8 S. Julia, M. Julia, and P. Graffin, Bull. Soc. Chim. France 1964, 3218.

9 J. P. Pete, Bull. Soc. Chim. France 1967, 357.

10 W. G. Dauben and G. H. Berezin, J. Amer. Chem. Soc. 89, 3449 [1967].

11 C. H. Heathcock and S. R. Poulter, J. Amer. Chem. Soc. 90, 3766 [1968].

12 M. J. Jorgenson and T. Leung, J. Amer. Chem. Soc. 90, 3769 [1968].

13 R. M. Kellog and J. Buter, J. Org. Chem. 36, 2236 [1971].

14 A. Y. Mayer, B. Muel, and Kasha, J. Mol. Spectroscopy 43, 262 [1972].

15 J. A. Al-khafaji and M. Shanshal, Z. Naturforsch. 30 a, 1023 [1975].

$16 \mathrm{H}$. Günther and D. Wendisch, Angew. Chem. 78, 266 [1966] ; ibid. 78, 544 [1966].
17 A. Streitwieser Jr., Molecular Orbital Theory for Organic Chemistry, J. Wiley and Sons Inc., 1961. See also R. S. Mulliken, C. A. Rieke, and W. G. Brown, J. Amer. Chem. Soc. 63, 41 [1941].

18 A similar treatment for the propen molecule was recently published by L. Libit and R. Hoffmann, J. Amer. Chem. Soc. 96, 1370 [1974].

19 For references see Ref. ${ }^{14}$ and ${ }^{15}$.

20 R. Gleiter, J. A. Al-Khafaji, and M. Shanshal, to be published.

21 J. A. Al-Khafaji, M. Sc. Thesis, University of Baghdad 1974.

22 J. F. Music and F. A. Matsen, J. Amer. Chem. Soc. 72, 5256 [1950].

${ }^{23}$ F. Effenberger and W. Kurtz, Chem. Ber. 106, 511 [1973]. 